\title{
Interferometric resolution boosting for spectrographs
}

\author{
D.J. Erskine and J. Edelstein
}

This article was submitted to

SPIE Conference on Astronomical Instrumentation, Glasgow, United Kingdom, June 21-25, 2004

\section{June 28, 2004}

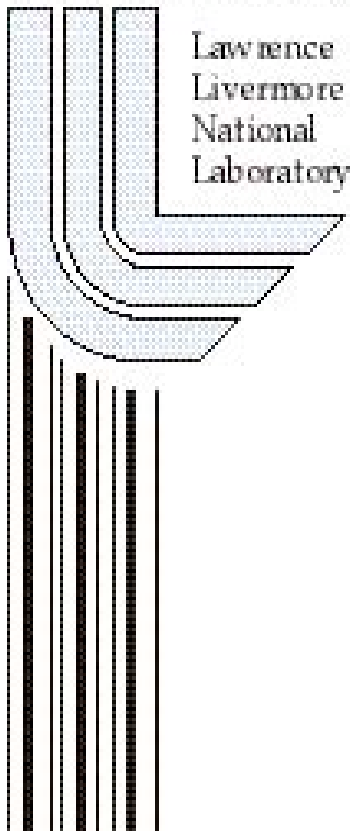


This document was prepared as an account of work sponsored by an agency of the United States Government. Neither the United States Government nor the University of California nor any of their employees, makes any warranty, express or implied, or assumes any legal liability or responsibility for the accuracy, completeness, or usefulness of any information, apparatus, product, or process disclosed, or represents that its use would not infringe privately owned rights. Reference herein to any specific commercial product, process, or service by trade name, trademark, manufacturer, or otherwise, does not necessarily constitute or imply its endorsement, recommendation, or favoring by the United States Government or the University of California. The views and opinions of authors expressed herein do not necessarily state or reflect those of the United States Government or the University of California, and shall not be used for advertising or product endorsement purposes.

This work was performed under the auspices of the U.S. Department of Energy by University of California, Lawrence Livermore National Laboratory under Contract W-7405-Eng-48.

Available from

Lawrence Livermore National Laboratory

Technical Information Department's Digital Library

http://www.llnl.gov/library/ 
UCRL-PROC-204704

\title{
Interferometric resolution boosting for spectrographs
}

\author{
David J. Erskine ${ }^{a}$ and Jerry Edelstein ${ }^{b}$ \\ ${ }^{a}$ Lawrence Livermore Nat. Lab., 7000 East Ave, Livermore, CA 94550 \\ ${ }^{b}$ Space Sciences Lab. at Univ. of Calif., Berkeley, CA 94720-7450
}

\begin{abstract}
Externally dispersed interferometry (EDI) is a technique for enhancing the performance of spectrographs for wide bandwidth high resolution spectroscopy and Doppler radial velocimetry. By placing a small angle-independent interferometer near the slit of a spectrograph, periodic fiducials are embedded on the recorded spectrum. The multiplication of the stellar spectrum times the sinusoidal fiducial net creates a moiré pattern, which manifests high detailed spectral information heterodyned down to detectably low spatial frequencies. The latter can more accurately survive the blurring, distortions and CCD Nyquist limitations of the spectrograph. Hence lower resolution spectrographs can be used to perform high resolution spectroscopy and radial velocimetry. Previous demonstrations of $\sim 2.5 \times$ resolution boost used an interferometer having a single fixed delay. We report new data indicating $\sim 6 \times$ Gaussian resolution boost (140,000 from a spectrograph with 25,000 native resolving power), taken by using multiple exposures at widely different interferometer delays.
\end{abstract}

Keywords: externally dispersed interferometer, EDI, interferometry, Fourier transform spectrometer, high resolution spectroscopy, Doppler, radial velocity

\section{INTRODUCTION}

An externally dispersed interferometer (EDI) is the series combination of an angle-independent Michelson interferometer ${ }^{1}$ with an external grating spectrograph (Fig. 1). The interferometer has a nonzero delay $(\tau)$ or optical path length difference, typically 1 to $3 \mathrm{~cm}$, that creates a sinusoidal frequency dependence to its transmission. Inclusion of the interferometer into the light beam, like a filter, embeds (multiplies) a comb of very periodic sinusoidal fiducials on the input stellar spectrum, over the entire spectrograph bandwidth.

Through a heterodyning effect between the fiducials and stellar spectrum, high resolution spectral information forms broader moiré patterns which better survive spectrograph blurring and resist instrumental drifts, allowing use of lower resolution spectrographs to perform precision Doppler radial velocimetry and spectroscopy normally restricted to higher resolution instruments. Data reduction arithmetically separates fringing from ordinary spectra components, and the conventional spectrum is obtained "for free". (For example, summing the complementary outputs always returns the ordinary spectrum.) Assuming a lossless interferometer that uses both complementary outputs, the EDI can not only provide the conventional information, but also new information in the fringing component. Thus logically the EDI can only increase the net information measured, per detected photon. This remarkable claim is consistent with an apparent increase in the coherence length of a grating as viewed through an interferometer (Fig. 2) when the interferometer delay is similar to or larger than the native grating coherence length. A more visceral demonstration is provided by Fig. 3 which shows a graphical manifestation of heterodyning, also called a moiré effect.

\subsection{Variety of applications}

The EDI was originally developed for radial velocimetry (under a Doppler shift the entire moire pattern shifts in phase). However the EDI's ability to measure precise $(\sim \lambda / 20,000)$ white light fringe shifts between simultaneous multiple spectral sources suggests additional metrology technologies. The EDI has been proposed ${ }^{2}$ to be used with a long baseline interferometer "front end" to measure precision angular differences between simultaneous multiple star targets. Because this would be a differential measurement using the same optical path and CCD pixels simultaneously for each star, it

Further author information:

D.J.E.: E-mail: erskine1@1lnl.gov, Telephone: 19254229545

J.E.: E-mail: jerrye@ssl.berkeley.edu, Telephone: 15106420599 

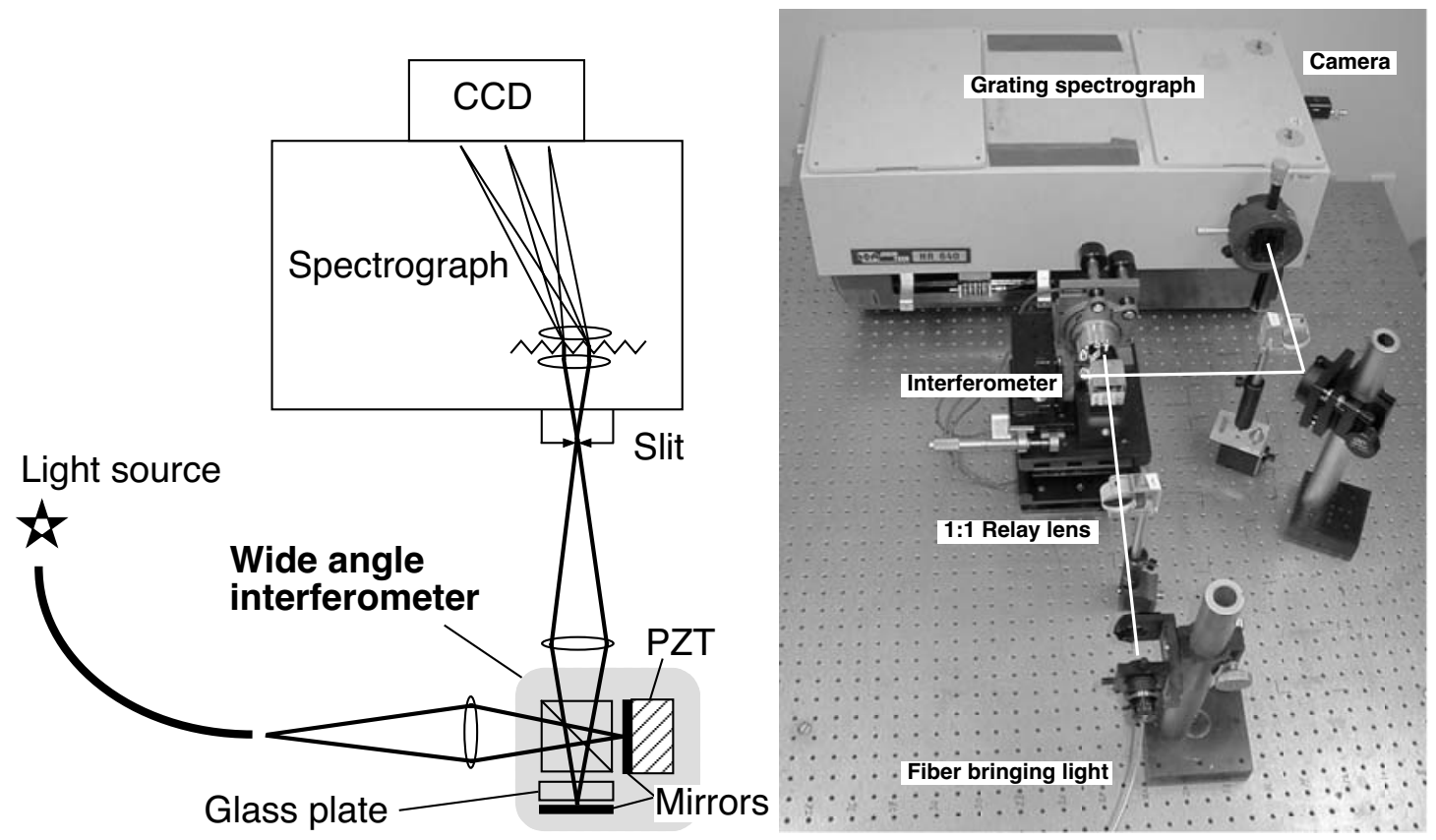

Figure 1. Schematic and photograph of EDI apparatus used to demonstrate $\sim 6 \times$ resolution boosting. The light to be measured enters via fiber at bottom of photo. For the ordinary technique the light would go directly into the Jobin-Yvon 640 grating spectrograph. For the EDI technique an interferometer "filter" having a nonzero delay is placed into the beam. This overlays sinusoidal fringes vs wavelength on the input spectrum, which creates moiré patterns. The end of the fiber is imaged to the interferometer mirror plane, which in turn is imaged to the spectrograph slit plane. A glass etalon next to one of the interferometer mirrors contributes to the delay value, and provides angular independence to the delay so that wide fibers can be used. Data was taken sequentially with a series of etalon thicknesses. A single delay can produce a resolution boost of $\sim 2.5 \times$. Multiple delays allows much larger boosts, proportional to the thickest delay used.

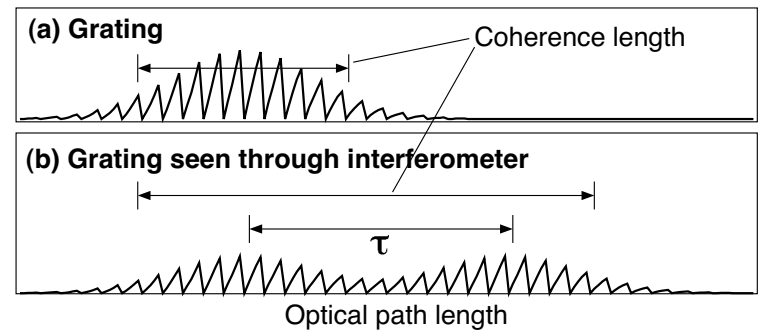

Figure 2. Apparent increase in coherence length of a grating when viewed through an interferometer having delay $\tau$. Any object viewed through a Michelson interferometer appears twice (with $50 \%$ intensity for each image), and with the second image deeper (delayed). The two images of the grating appear as a single grating with a longer coherence length. Since the spectral resolution is proportional to the net grating coherence length, the resolution increases. 


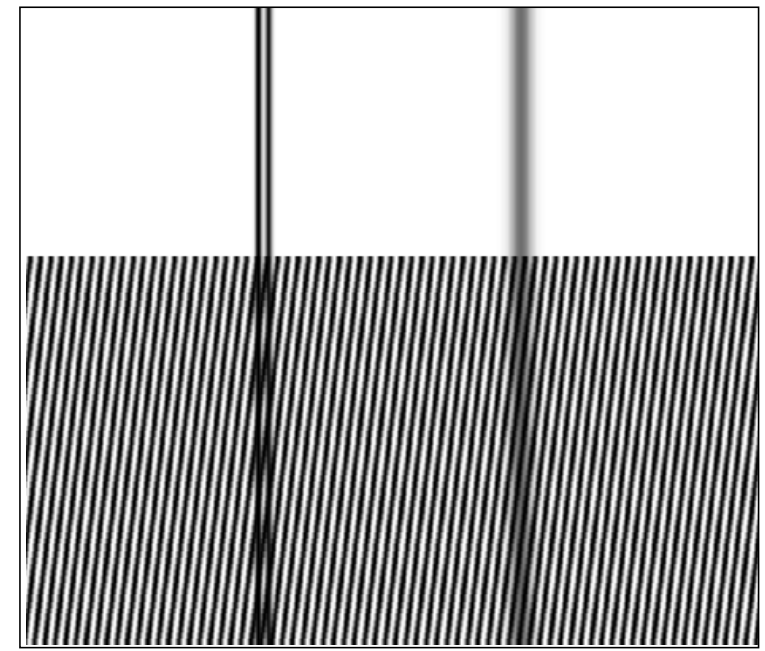

Figure 3. Graphical demonstration of the increased resolving power coming from a heterodyning effect, which generates moiré patterns. If viewed from a distance, the doublet of lines on the left in the upper panel appears indistinguishable from the single line on the right. On the bottom panel a sinusoidal pattern is superimposed, representing the interferometer transmission vs wavelength or frequency. The presence of the generated moiré pattern immediately distinguishes the doublet. Hence the resolution has increased.

is insensitive to microscopic drifts in the optical path length or interferometer baseline, a challenging problem for the conventional astrometry technique.

More recently, the EDI's has been applied to high resolution spectroscopy, either as a compact stand-alone instrument, or as performance boosting filter or insert to an existing spectrograph facility. This application is the focus of this article. Essentially, we have "eyeglasses" that can be inserted into the beam path of any spectrograph to boost its spectral resolution by a factor of $2-6 \times$ or more. The EDI can resolve features beyond the Nyquist limit of the CCD, because the moiré patterns encoding the high resolution information are broader than the CCD pixel spacing. The EDI also improves spectrograph stability because the broader moire patterns are less susceptible to changes in shape of the light beam at the slit, aberrations of spectrograph optics, and mechanical drifts of the CCD.

\subsection{Comparison to other techniques}

The EDI has a simultaneous bandwidth advantage over internally dispersed interferometers ${ }^{3,4}$ (Holographic Heterodyning Spectrograph, Spatial Heterodyning Spectrograph) which have bandwidth limitations due to rapid change in fringe periodicity vs wavelength. In comparison with a dispersed Fabry-Perot interferometer producing narrow impulse spectral fiducial fringes, ${ }^{5}$ EDI's sinusoidal fiducial fringes transmit greater average flux, provide a heterodyning effect, and allow elegant trigonometric recovery of precise spectral information from as few as three phase-stepped data recordings.

The EDI is mathematically related to techniques that combine a Fourier Transform Spectrometer (FTS) with a disperser. $^{6,7}$ The EDI approach uses massively $(>1000)$ parallel spectral channels with a fixed or few fixed delays. The dispersed FTS approach uses many more delay channels (by scanning the delay) with usually fewer spectral channels. We predict that the photon signal to noise ratio for spectroscopy is proportional to the square root of the number of simultaneous dispersed spectral channels that can be measured at a given delay value, and thus will favor EDI. The EDI using an echelle spectrograph (i.e. $\sim 10^{4}$ channels) has $\sim 100 \times$ better photon signal to noise ratio than an undispersed FTS over the optical bandwidth, but yet retains much of the instrument lineshape stability for which FTS is valued.

\subsection{Some literature}

The EDI demonstrated $1 \mathrm{~m} / \mathrm{s}$ scale Doppler precision in 1999 in benchtop measurements on laboratory sources. ${ }^{8}$ The first EDI stellar radial velocimetry measurements were performed ${ }^{9,10}$ at the Lick Observatory $1-\mathrm{m}$ in 1999 and have a few $\mathrm{m} / \mathrm{s}$ precision. The EDI has been used to detect the exoplanet around 51 Pegasi at Kitt Peak. ${ }^{11}$ 
The EDI instrument theory has been described. ${ }^{12,13}$ Reference 13 is comprehensive and provides a theoretical foundation for the heterodyning effect used in the resolution boosting application, and describes both uniform and transversely splayed phase along the spectrograph slit, i.e. can be applied to echelle gratings and 1-D imaging spectrographs, as well as linear gratings. Spectral resolution boosting has been demonstrated ${ }^{14,15}$ for echelle and linear grating spectrographs in both laboratory and observatory settings.

\section{THEORY: CONVENTIONAL SPECTROSCOPY}

The conventional detected spectrum, $B_{\text {ord }}(\nu)$, is the convolution of the input spectrum, $S_{0}(\nu)$, and the spectrograph line spread function $\operatorname{LSF}(\nu)$,

$$
B_{\text {ord }}(\nu)=S_{0}(\nu) \otimes L S F(\nu)
$$

where frequency is $\nu=1 / \lambda$ in units of $\mathrm{cm}^{-1}$. The full width at half max (FWHM) of $L S F(\nu)$ is $\Delta \nu$ and is related to resolution (resolving power) by $R=\nu / \Delta \nu$. The convolution of Eq. 1 is conveniently expressed in Fourier-space,

$$
b_{\text {ord }}(\rho)=s_{0}(\rho) l s f(\rho),
$$

where lower case symbols are the transformed versions, and $\rho$ is the spatial frequency along the dispersion axis in cycles per $\mathrm{cm}^{-1}$. The $l s f(\rho)$ is thus the transfer function of the impulse response $\operatorname{LSF}(\nu)$.

\section{THEORY: EDI SPECTROSCOPY}

In an ideal EDI both complementary outputs are dispersed and detected (necessarily different CCD pixels), so that the net flux is not reduced by interferometer insertion. Then it is appropriate to use the normalized interferometer transmission $T^{\prime}(\nu)$, which is a sinusoidal spectral comb

$$
T^{\prime}(\nu)=1+\gamma \cos (2 \pi \tau \nu+\phi)
$$

where $\gamma$ is the interferometer visibility, assumed unity for now, and $\tau$ is the nominal interferometer delay in units of $\mathrm{cm}$. Three or four spectra $B_{\phi}$ are recorded at phase values $\phi$ differing by $120^{\circ}$ or $90^{\circ}$, respectively, and designated $B_{0}, B_{90}$, etc., so that the phases are distributed evenly around the circle. The $\phi$ is created by changes in $\tau, \phi=2 \pi \Delta \tau$.

The passage of light through the interferometer multiplies the spectral comb $T^{\prime}(\nu)$ with the input spectrum prior to any blurring action from the external grating spectrograph. Hence the EDI detected signal is

$$
B_{\phi}(\nu)=\left[S_{0}(\nu) T^{\prime}(\nu)\right] \otimes L S F(\nu) .
$$

Equation 4 is re-expressed as a sum of the ordinary spectrum plus two complex counter-rotating fringing terms

$$
\begin{aligned}
B_{\phi}(\nu)= & B_{\text {ord }}(\nu)+\frac{1}{2}\left[S_{0}(\nu) e^{i \phi} e^{i 2 \pi \tau \nu}+\right. \\
& \left.S_{0}(\nu) e^{-i \phi} e^{-i 2 \pi \tau \nu}\right] \otimes L S F(\nu) .
\end{aligned}
$$

One needs to isolate a single fringing component. To do this, we form a linear combination of data which have been numerically rotated in synchrony with each exposure's phase. This creates a complex (or vector) spectrum called a "whirl”, W $(\nu)$. For N-recordings ( $\phi$ evenly spaced)

$$
\mathbf{W}(\nu)=\frac{1}{N} \sum B_{\phi} e^{i \phi}
$$

and specifically for four exposures every $90^{\circ}$

$$
\mathbf{W}(\nu)=\frac{1}{4}\left[\left(B_{0}-B_{180}\right)+i\left(B_{90}-B_{270}\right)\right] .
$$

Applying Eq. 6 or 7 to Eq. 5 we get

$$
\mathbf{W}(\nu)=\frac{1}{2}\left[e^{i 2 \pi \tau \nu} S_{0}(\nu)\right] \otimes L S F(\nu) .
$$




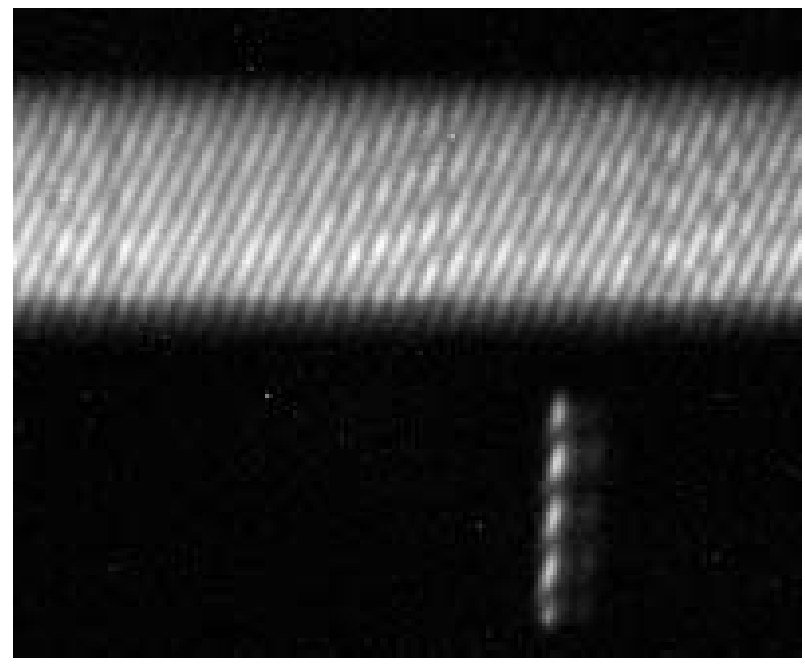

Figure 4. Raw CCD data for glass thickness of $1 / 4$ inch $(\tau=1.1 \mathrm{~cm})$ showing fringing spectra of iodine and $\mathrm{Hg}$ lamp (546 $\mathrm{nm}$ line) recorded in parallel through the same interferometer. Only 200 horizontal pixels shown, of 2500. Note spectrograph is imperfect, as seen in slant of $\mathrm{Hg}$ line and echos to its right. The spectrograph idiosyncrasies are divided out of the data during the concatenation process, and the composite EDI instrument response is dramatically more regular and stable to spectrograph point spread function irregularities.

The Fourier transform of the whirl is

$$
\mathbf{w}(\rho)=\frac{1}{2} \gamma s_{0}(\rho+\tau) \operatorname{lsf}(\rho)
$$

where we include the interferometer visibility $(\gamma)$ previously taken as unity.

This important equation describes the EDI formation of moiré fringes, a heterodyning effect expressed in the $s_{0}(\rho+\tau)$ argument. Fine spectral details having high $\rho$ are heterodyned (shifted by $\tau$ ) to measurable low $\rho$ prior to any blurring by the spectrograph's line spread function.

The ordinary spectrum is obtained from fringing spectra by summing several phase stepped exposures so that the fringing terms cancel,

$$
B_{\text {ord }}(\nu)=\frac{1}{N} \sum B_{\phi}
$$

or specifically for four recordings

$$
B_{\text {ord }}(\nu)=\frac{1}{4}\left(B_{0}+B_{180}+B_{90}+B_{270}\right) .
$$

Hence both ordinary and fringing spectra are obtained from the same data set. In this sense the EDI's fiducials are "transparent". Note that the fiducials are removed by summing the phase stepped exposures, instead of dividing by a fiducial spectrum, avoiding the potential increased noise from divide-by-zero issues.

The heterodyning is reversed by Fourier transforming the data from $\mathbf{W}(\nu)$ to $\mathbf{w}(\rho)$, translating it by $\tau$, and then inverse Fourier transforming it back to $\nu$-space. This procedure is described in more detail in Ref. 13.

\section{ON-GOING WORK: MULTIPLE DELAY EDI}

We have conducted in laboratory tests a preliminary demonstration of $\sim 6 \times$ resolution boosting using the multiple-delay EDI shown in Fig. 1. A native resolution of $\sim 25,000$ was boosted to a Gaussian lineshape resolution of $\sim 147,000$ over the entire bandwidth $(100 \AA)$ of the spectrograph. The input spectrum was provided by an iodine vapor cell backlit by an incandescent lamp.

We measured fringing spectra (such as Fig. 4) with a variety of fixed $\tau$ up to $4.5 \mathrm{~cm}$, by inserting into the interferometer glass etalons of various thicknesses up to 1 inch. For each etalon the distances of the interferometer mirrors were translated to re-establish the angle-independent condition whereby the virtual image of one mirror seen through the etalon 

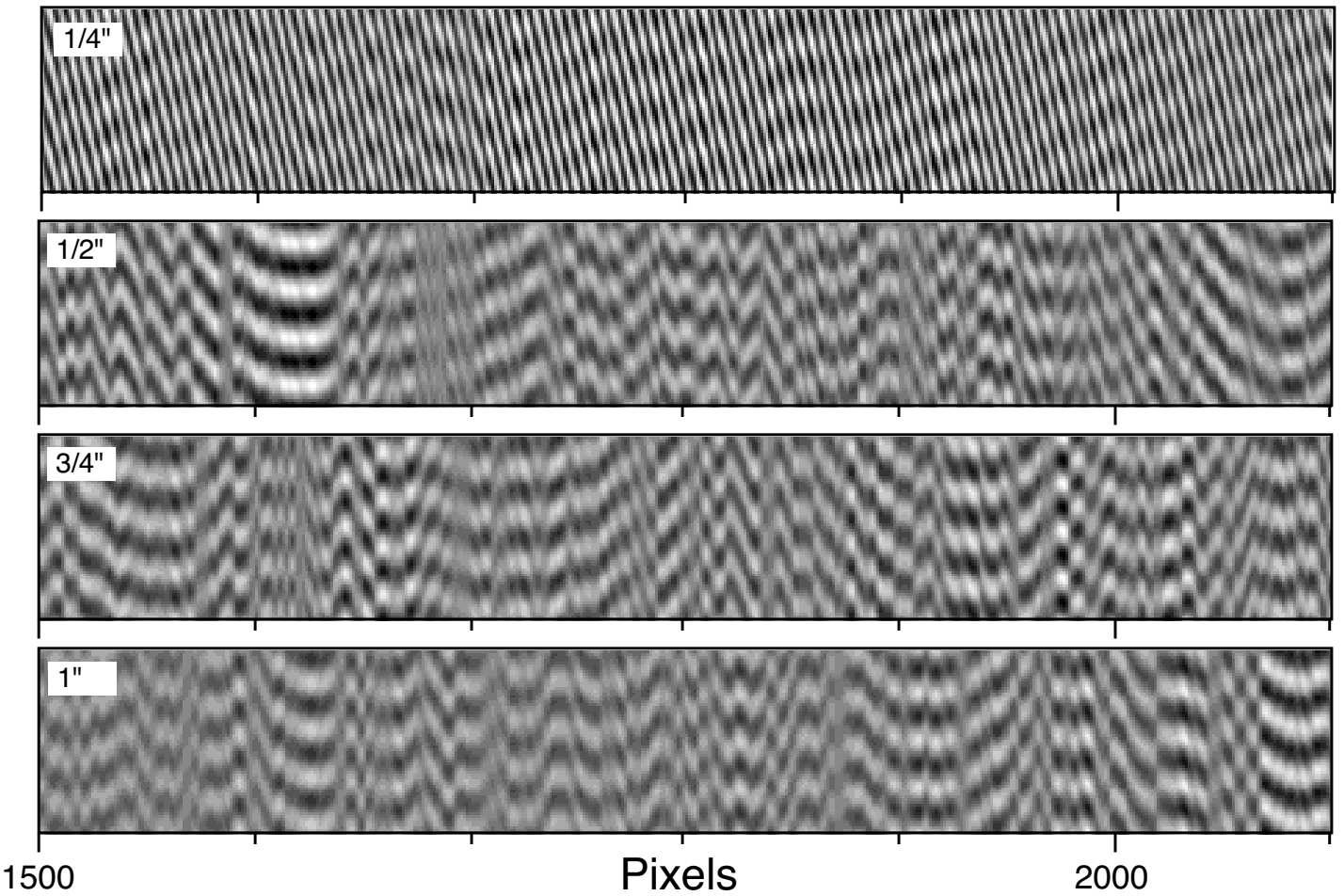

Figure 5. Measured moiré patterns for the iodine spectrum, for several interferometer delays (labeled by delay glass thickness). The nonfringing signal portions have been removed. As the glass thickness increases the interferometer transmission sinusoid becomes finer. (For delays above 1/4" it is too fine to be resolved.) During data analysis, the heterodyning that creates these patterns is reversed to deduce structure of the input spectrum at different spatial scales set by the sinusoid period.

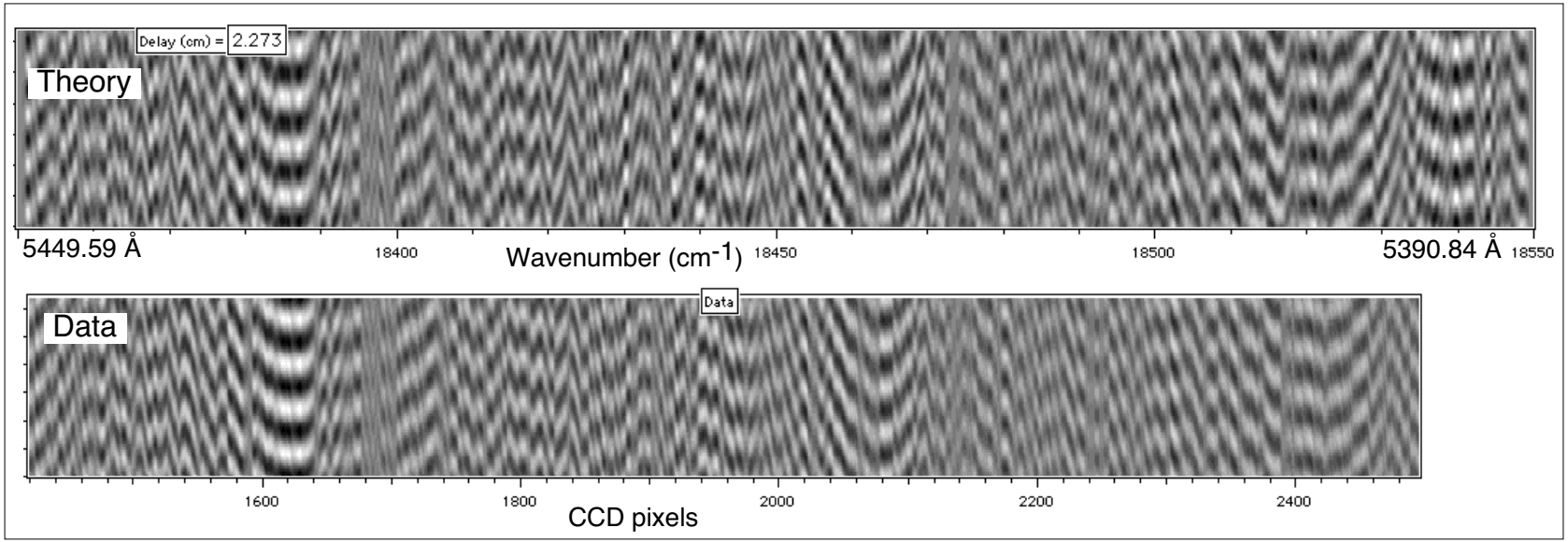

Figure 6. An example measured EDI data (bottom panel) for the $1 / 2$ inch thick glass agrees well with calculated moiré pattern (top panel), computed from a textbook iodine spectrum (from NOAO Kitt Peak FTS) by multiplying by a sinusoid of $1 /(2.273 \mathrm{~cm})$ periodicity, and blurring it. The ordinary spectrum (nonfringing) component has been removed. Both data and theory are shown in pseudofringe style, which is a method of displaying complex spectra where the phase and amplitude of the fringe at each wavelength channel is represented by a sinusoidal intensity pattern splayed vertically. Similarly for Fig. 5 . 


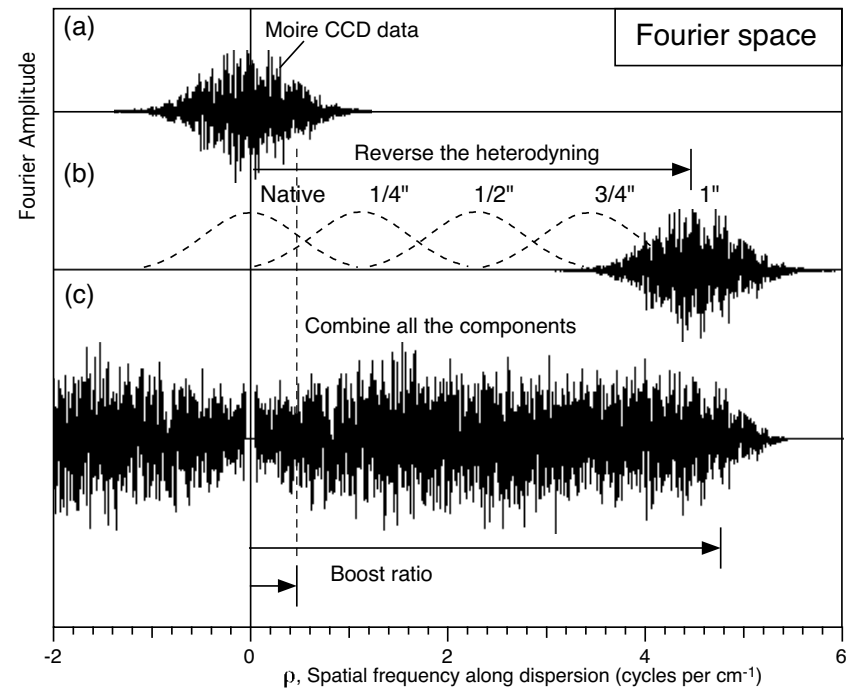

Figure 7. Steps in reconstructing a spectrum to higher resolution, illustrated in spatial frequency space, $\rho$. Note that $\rho$ units of cycles per $\mathrm{cm}^{-1}$ are same as delay, i.e. $\mathrm{cm}$. (a) The raw data recorded on CCD has a range of spatial frequencies limited by blurring of grating spectrograph. The ordinary spectrum is removed leaving the moire (fringing) component. (b) The spatial frequency of the data is shifted to its original higher spatial frequency by amount $\tau$, the interferometer delay. (c) Results from different delays are concatenated together to form a composite result. Taking the real part of this effectively copies the positive to negative branch. The composite result has a spatial frequency range that is much wider than the grating alone (dashed peak "Native"). Hence the spectral resolution has been boosted by a significant factor.

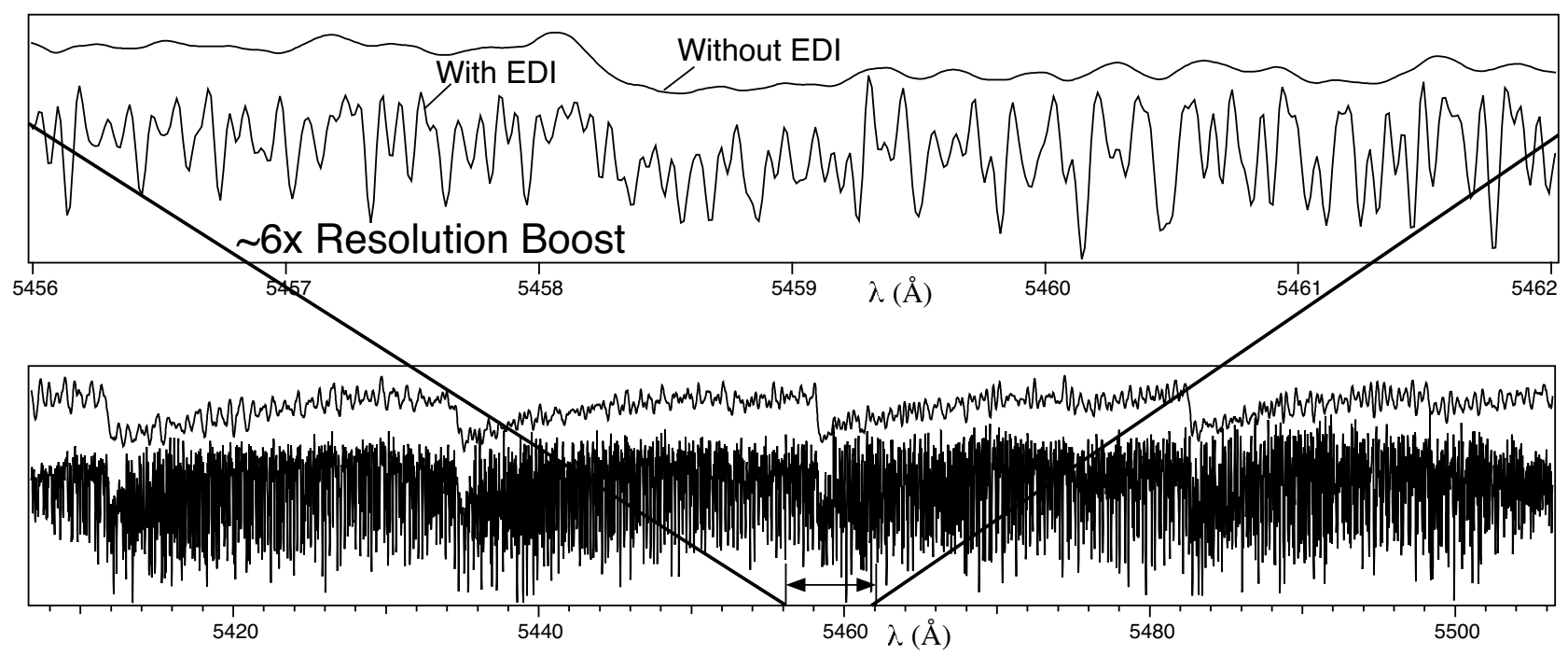

Figure 8. Laboratory EDI demonstration of a $\sim 6 \times$ resolution boosting effect measuring the iodine spectrum. Top curve is the conventional result, i.e. using the grating spectrograph by itself. The fine iodine lines cannot be seen. Inserting the interferometer prior to the spectrograph and postprocessing the data reveals these features (bottom curve) quite dramatically. This is the concatenated output having all spatial frequencies up to $5 \mathrm{~cm}$ uniformly detected. The native $\sim 25,000$ grating resolution was effectively boosted to $\sim 147,000$ Gaussian resolution. Theoretical spectrum at $\sim 370,000$ resolution is bottom curve. The bandwidth is the same as the native bandwidth of the grating spectrograph, in this case $\sim 100 \AA$. 
longitudinally superimposes the mirror of the other arm. Under this condition a glass thickness of 1 inch produced a delay of $4.5 \mathrm{~cm}$, measured spectroscopically, and proportionately for smaller thickness. Total glass thickness was stepped in increments of $1 / 8$ inch for thoroughness. In hindsight, four delays of $1 / 4$ inch increment (producing $\Delta \tau=1.1 \mathrm{~cm}$ ) is sufficient to cover the delay range shoulder to shoulder from 0 to $4.5 \mathrm{~cm}$ for the native spectrograph resolution of 25,000 .

Figure 5 shows processed fringing data measured at different delays. The data has had the ordinary nonfringing spectrum component removed to reveal the moire patterns. These patterns contain spatial frequencies limited by the spectrograph blurring. The native spectrograph response when plotted vs $\rho$ (i.e. a modulation transfer function) is a peak having half width half max (HWHM) of $\sim 0.6$ cycles per $\mathrm{cm}^{-1}$. The relationship between resolution and the HWHM spatial frequency $\left(\rho_{h w h m}\right)$ for a Gaussian instrument response is

$$
R=\frac{\nu 2 \rho_{h w h m}}{0.88}
$$

(The $\nu$ and $\rho$ are a Fourier transform pair, and for a Gaussian shape the full widths at half max (FWHM) follow an uncertainty principle: $\Delta \rho \Delta \nu=(4 \log 2 / \pi)=0.8825$.

To recover the high spatial frequency information, the moiré data is upshifted in $\rho$ by amount $\tau$. This process is depicted in Fig. 7. For the 1 inch glass case, the moire pattern is Fourier transformed, shifted to the left by $4.5 \mathrm{~cm}$, and then inverse Fourier transformed. The signal is then forced to be real valued by adding its complex conjugate.

The delay value $\tau$ can be found for small $\tau$ by counting the number of fringes in the continuum between two known wavelengths. For larger $\tau$ beyond the Nyquist limit of the CCD $(3 \mathrm{~cm}), \tau$ can be found by fitting measured moiré data to calculated patterns using a textbook iodine spectrum provided by Kitt Peak NOAO, measured by an FTS at 400,000 resolution. These are calculated by multiplying a sinusoid having an adjustable period $(1 / \tau)$ against the reference spectrum, and then low pass filtering the product to reveal the moiré. Figure 6 shows the excellent agreement between theoretical and measured moiré patterns for the case of $1 / 2$ inch glass $(2.273 \mathrm{~cm}$ delay).

Data from each delay value contributed a different set of spatial frequencies, centered at $\tau$, that were concatenated together to form the net output, which spans from 0 to $5 \mathrm{~cm}$. (Note that spatial frequency and delay have same units, cycles per $\mathrm{cm}^{-1}$ or $\mathrm{cm}$ ). Equalization was used to smooth the bumps, so that the net transfer function of the EDI process was approximately flat from zero to the maximum delay used. In the concatenation process the ordinary spectrum provides the lowest spatial frequency range $0-0.6 \mathrm{~cm}$.

Figure 8 (bottom curve) shows the measured composited EDI spectrum of iodine. The Figure compares the conventional spectrum (upper curve) with that using the interferometer (lower curve). The improvement in resolving power is dramatic. Many fine spectral lines that are unresolved by the conventional spectrograph are now fully resolved using the EDI. This resolution boosting effect occurs over the full bandwidth of the spectrograph (lower panel), greatly increasing the total information returned by the measurement.

\subsection{Data taking}

Data was taken in the "slanted fringe" mode (multi-phase) by tilting an interferometer mirror so that the fringe phase $(\phi)$ varies linearly along the slit length. In this mode it is easy to maximize the moire visibility while watching a TV grade $\mathrm{CCD}$ and adjusting the longitudinal position of an interferometer mirror. Since all phases are present at once in this mode, at different positions $(Y)$ transverse to the dispersion axis, only a single exposure is required per delay. Multiple exposures were taken per etalon, by stepping the overall interferometer phase by moving a PZT-mounted interferometer mirror in a piston manner. This has the advantage of eliminating any fixed pattern errors, such as those due to imperfect flatfielding of the CCD or imperfect normalization of the beam intensity profile along the slit. The conversion of slanted fringe raw CCD data into a complex spectrum, and piston phase stepping data reduction are described in section 8 of Ref. 13 .

An Hg lamp was recorded simultaneously with the iodine on a neighboring section of the slit passing through the same interferometer. This indicates spectrograph instrument response and interferometer phase. A HeNe laser passing through the interferometer whose fringes where monitored on a TV camera was used to determine instantaneous interferometer phase. Since the exposures were short $(10 \mathrm{~s})$ active cavity stabilization was not needed. Piston phase stepping was performed by advancing the HeNe fringe by $1 / 4$ wave. Since the HeNe wavelength $(633 \mathrm{~nm})$ differs $16 \%$ from the approximate wavelength $(\sim 546 \mathrm{~nm})$ of the recorded spectra the phase steps for the data were not $90^{\circ}$. We have written custom algorithms which successfully reduce the data in spite of irregular phase steps. This issue also pertains to echelle spectrographs where the wide bandwidth $(400-900 \mathrm{~nm})$ precludes constant $\phi$ vs $\lambda$. 


\subsection{Boost ratio}

One method of defining the net boosting factor obtained is the ratio $\left(Z_{\rho}\right)$ of the highest spatial frequency reasonably detectable with and without the interferometer, measured at the local HWHM. This would be $5 \mathrm{~cm}$ (i.e. $\tau+0.6)$ divided by $0.6 \mathrm{~cm}$, producing $Z_{\rho} \approx 8$ of boost. This $Z$ definition is independent of equalization.

Another definition is the ratio $\left(Z_{G}\right)$ of instrument linewidths when the EDI instrument lineshape is equalized to be Gaussian (which has minimal ringing) by attenuating the higher $\rho$ data. In going from a rectangular to Gaussian modulation transfer function shape, the HWHM decreases to $70 \%$, so $Z_{G} \approx 0.7 Z_{\rho} \approx 6$. (Note that during this equalization process the noise at high spatial frequencies, as well as the signal, is reduced. Hence the signal to noise properties are more represented by $Z_{\rho}$ than by the $Z_{G}$.) The EDI resolution is independent of spectrograph resolution, and is proportional to the maximum delay $\left(\tau_{\max }\right)$ used. For a Gaussian equalization is used, then $\rho_{h w h m} \sim 0.7 \tau_{\max }$ and thus

$$
R=\frac{\nu 2(0.7)}{0.88} \tau_{\max }
$$

\section{CONCLUSIONS}

By inserting an interferometer in series with a grating spectrograph and postprocessing the moire patterns formed in the fringing spectra, one can increase the effective resolving power of the instrument. We have made a preliminary demonstration that resolution can be boosted by at least a factor of 6 by the use of multiple interferometer delays. This is an improvement over the single delay boosting of $\sim 2.5 \times$ demonstrated $^{15}$ on echelle and linear gratings. The spatial frequency along the dispersion direction $(\rho)$ being detected for the highest delays $(4.5 \mathrm{~cm})$ are beyond the Nyquist limit of the CCD, which is $3 \mathrm{~cm}$ (or cycles per $\mathrm{cm}^{-1}$ ). This is due to a heterodyning effect which shifts high spatial frequencies to lower values detectable by the spectrograph and CCD.

We expect to obtain $\sim 12 \times$ Gaussian resolution boost by repeating the above measurements with the spectrograph slit widened to produce 12,500 native resolving power. This will require data measured at eight delay values, every $1 / 8$ inch up to 1 inch of glass thickness. We have identified an interferometer design which measures multiple delays simultaneously at different positions along the long slit. This parallel configuration would produce a snapshot spectrograph capable of measuring high speed single shot phenomenon, something which a classic FTS having a scanning delay cannot do. The EDI preserves imaging information, and could be used to measure many targets along the spectrograph slit simultaneously. The noise and effective instrument lineshape properties of EDI are being investigated. Full pass interferometer designs such as the Mach-Zehnder that more easily direct both outputs to the spectrograph have been identified.

\section{ACKNOWLEDGMENTS}

Reference iodine spectra were provided by Kitt Peak/NOAO. Funds provided by the Laboratory Directed Research and Development Program. This work was performed under the auspices of the U.S. Department of Energy by the University of California, Lawrence Livermore National Laboratory under contract No. W-7405-Eng-48.

\section{REFERENCES}

1. R. Hilliard and G. Shepherd, "Wide-angle Michelson for measuring doppler line widths," J. Opt. Soc. Am.56, p. 362, 1966.

2. D. Erskine and J. Edelstein, "Spectral astrometry mission for planets detection," in Interferometry in Space, ed. M. Shao, pp. 695-706, SPIE Proc. 4852, Feb. 2003.

3. N. Douglas, "Heterodyned holographic spectroscopy," PASP 109, p. 151, 1997.

4. J. Harlander, R. Reynolds, and F. Roesler, "Spatial heterodyne spectroscopy for the exploration of diffuse interstellar emission lines at far-ultraviolet wavelengths," ApJ 396, p. 730, 1992.

5. R. S. McMillan, T. L. Moore, M. L. Perry, and P. H. Smith, "Radial velocity observations of the sun at night," ApJ 403, pp. 801-809, Feb. 1993.

6. B. Mosser, J. Maillard, and F. Bouchy, "Photon Noise-limited Doppler Asteroseismology with a Fourier Transform Seismometer. I. Fundamental Performances,” PASP 115, pp. 990-1001, Aug. 2003. 
7. A. R. Hajian, "A High Throughput Modified FTS - A New Direction in Astronomical Spectroscopy," Bulletin of the American Astronomical Society 30, pp. 859-+, May 1998.

8. D. Erskine and J. Ge, "Novel interferometer spectrometer for sensitive stellar radial velocimetry," in Imaging the Universe in Three Dimensions: Astrophysics with Advanced Multi-Wavelength Imaging Devices, W. van Breugel and J. Bland-Hawthorn, eds., ASP 195, p. 501, 2000.

9. J. Ge, D. Erskine, and M. Rushford, “An externally dispersed interferometer for sensitive doppler extra-solar planet searches," PASP 114, pp. 1016-1028, 2002.

10. J. Ge, "Erratum: Fixed delay interferometry for doppler extrasolar planet detection," ApJ 593, p. L147, 2003.

11. J. C. van Eyken, J. Ge, S. Mahadevan, and C. DeWitt, "First Planet Confirmation with a Dispersed Fixed-Delay Interferometer," ApJ 600, pp. L79-L82, Jan. 2004.

12. J. Ge, "Fixed delay interferometry for doppler extrasolar planet detection," ApJ 571, pp. L165-168, 2002.

13. D. Erskine, "An externally dispersed interferometer prototype for sensitive radial velocimetry: Theory and demonstration on sunlight," PASP 115, pp. 255-269, 2003.

14. D. Erskine and J. Edelstein, "High-resolution broadband spectral interferometry," in Future EUV/UV and Visible Space Astrophysics Missions and Instrumentation, ed. J. C. Blades, O. H. Siegmund, pp. 158-169, SPIE Proc. 4854, Feb. 2003.

15. D. Erskine, J. Edelstein, M. Feuerstein, and B. Welsh, "High Resolution Broadband Spectroscopy using an Externally Dispersed Interferometer,” ApJ 592, pp. L103-L106, 2003. 